\title{
Novel Control Strategy of Single Matrix Traction Converter - Variable Switching Frequency
}

\author{
Bedrich Bednar ${ }^{1}$, Vojtech Blahnik ${ }^{1}$, Pavel Drabek ${ }^{1}$, Martin Pittermann ${ }^{2}$ \\ ${ }^{1}$ Regional Innovation Centre for Electrical Engineering, University of West Bohemia, \\ Univerzitni 8, Pilsen 306 14, Czech Republic \\ ${ }^{2}$ Department of Electromechanics and Power Electronics, University of West Bohemia, \\ Univerzitni 8, Pilsen 306 14, Czech Republic \\ bead@kev.zcu.cz
}

\begin{abstract}
This paper describes research into a novel control strategy of traction converter for the AC-Trolley Line with the middle frequency transformer (MFT). Attention is paid to the problematics of finding a suitable control algorithm that will provide minimal magnetic saturation. This could be ensured by variable pulse widths, determined according to the actual value of input voltage. Special attention is focused on the single-phase matrix converter topology and the best commutation solution. Two basic methods of controlling the switches in the matrix converter are discussed. The first method requires some knowledge of the converter output current polarity and the second method assumes some knowledge of the input voltage polarity. In addition, the article describes the switching method in uncertain states - when the voltage or current cross zero. Moreover, the paper presents a new modular topology with several separate middle frequency transformers. The proposed algorithm is described and implemented in the laboratory model.
\end{abstract}

Index Terms-Matrix converters, electric vehicles, transformer cores, high-voltage techniques.

\section{INTRODUCTION}

This research has been motivated by our industrial partner's demand to reduce the weight of the electrical equipment of the railway vehicle (mainly the bulky line traction transformer [1] found on board of railway vehicles) in order to enhance rated power.

Figure 1 shows the basic topology of the classic AC trolley line traction vehicle. High voltage input is reduced by the traction transformer (TT). Due to very low supplying frequency (i.e. traction systems in Europe use trolley voltage $25 \mathrm{kV} / 50 \mathrm{~Hz}$ or $15 \mathrm{kV} / 16.7 \mathrm{~Hz}$ ), the traction transformer is very bulky (for example $15 \mathrm{t}$ for rated power $6.4 \mathrm{MW}$ ). The transformer's weight causes a lot of problems especially for total vehicle weight due to limited weigh per axle (22.5 tons/axle, i.e. 4-axle locomotive limitation is about 90 tons). The total weight limitation has a significant impact

Manuscript received January 9, 2015; accepted March 23, 2015

This research has been supported by the European Regional Development Fund and Ministry of Education, Youth and Sports of the Czech Republic under project No. CZ.1.05/2.1.00/03.0094: Regional Innovation Centre for Electrical Engineering (RICE) and project No. SGS-2015-038. on traction force in modern high-speed locomotives and special purpose vehicles (electric multiple units, tram-train for AC-Trolley Line etc.).

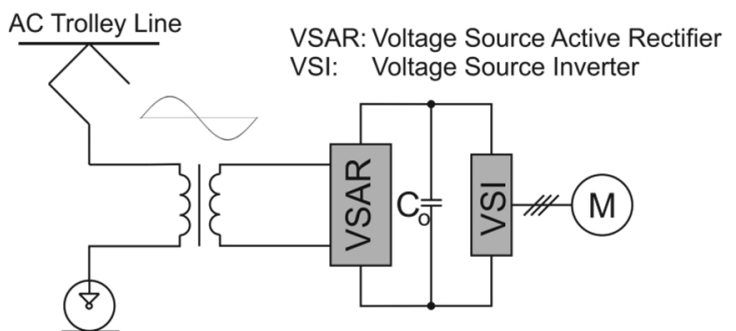

Fig. 1. Classical AC traction vehicle topology with line traction transformer.

The recent trend in research into new traction converter topologies for multi-system locomotives, trains, suburban units and vehicles supplied by AC electrification systems is strongly oriented towards the reduction of weight and emphasizes dimensions of a new generation of electrical equipment used in these vehicles [2]-[11]. The investigated traction converter configurations are often inspired by known topologies from switching power supplies, which are however, operated at dramatically different power levels. One of the perspective configurations of the new traction converters is a topology employing MFT (Fig. 2).

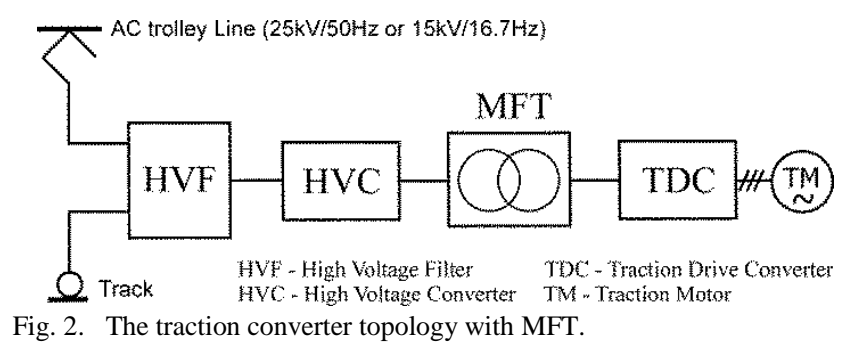

Increasing traction transformer frequency can be realized by implementing the special high-voltage input frequency converter (HVC) [2], [3], [6]-[11]. The HVC can be realised as a multi-level converter $\mathrm{M}^{2} \mathrm{LC}$ [3], serial connection of indirect frequency converters [7]-[10] or serial connection of direct frequency converters [11].

This paper deals with traction converter topology with 
MFT and serial connection of direct frequency converters. On the contrary, in this paper the HVC is based on matrix converters, comparing to [11], where the direct frequency converters operate as cycloconverters (there is not input filter $\mathrm{HVF}$ and therefore input converter HVC cannot operates as matrix converter).

Figure 3 shows the topology with the series connection of medium-voltage converters the primary side of the MFT and one converter on the secondary side of the MFT. Figure 3 presents only 2 levels on the primary side realised by the matrix converters MC [12]-[15]), however the real application will consist of a higher number of levels (for example 14 cells which use $6.5 \mathrm{kV}$ semiconductor elements on $25 \mathrm{kV}$ trolley line).

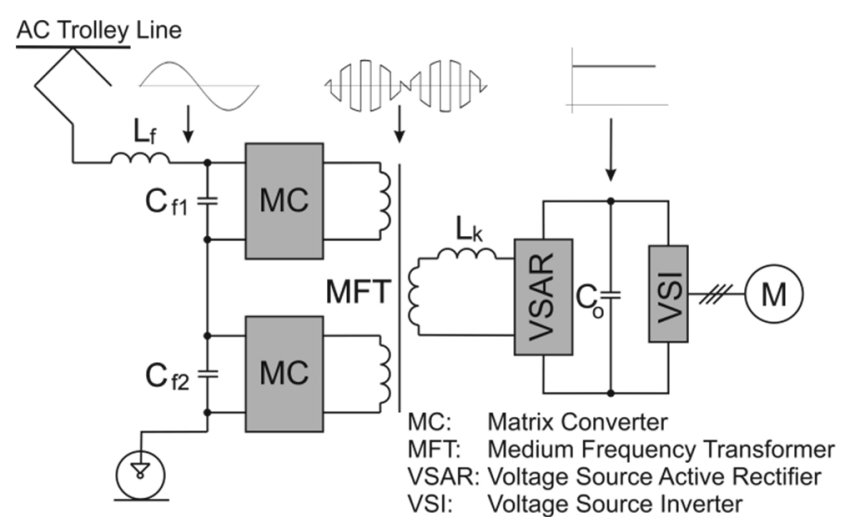

Fig. 3. Modern topology with middle frequency transformer (MFT) and matrix converters (MC).

The input trolley voltage is divided by the input filter (serial connection of capacitances $C_{f 1}, C_{f 2} \ldots$ ). For safe operation it is very important to spread the input voltage through these capacitances. The MCs transforms the $50 \mathrm{~Hz}$ (or $16.7 \mathrm{~Hz}$ ) sine-wave to $400 \mathrm{~Hz}$ rectangular signal (influenced by 2-order harmonic due to single phase AC trolley system).

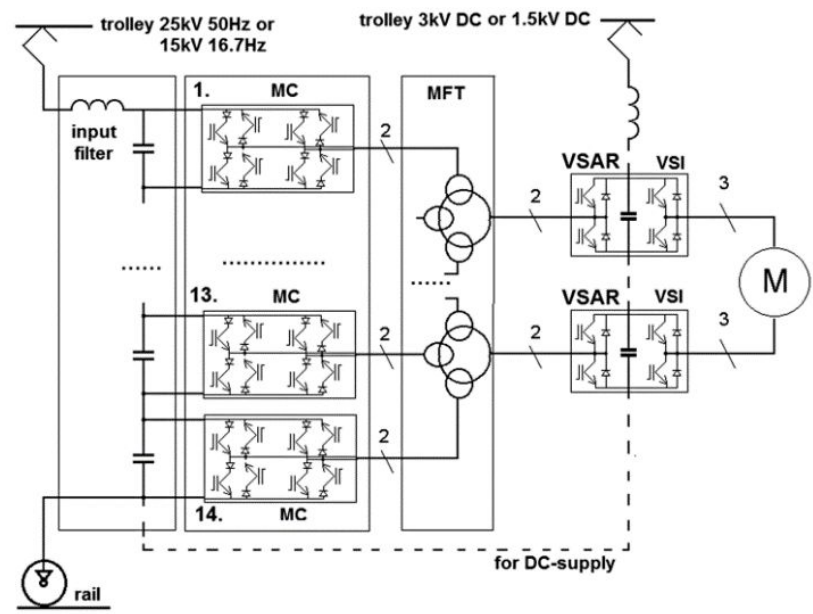

Fig. 4. Multisystem vehicle (for AC-Trolley Line and DC-Trolley Line).

The secondary side of the MFT is similar to the classic topology (Fig. 1) with respect to higher operational frequency. The output of the indirect frequency converter (with high value of capacitance $\mathrm{C}_{O}$ in DC-link) is used for filtering this low-frequency power ripple. Multisystem traction vehicle (DC system + AC system $50 \mathrm{~Hz}$ and $16.7 \mathrm{~Hz}$ ) can use capacitance $\mathrm{C}_{\mathrm{O}}$ as the input filter for DCtrolley. Figure 4 shows the power circuits of modern multisystem vehicles for all four traction systems used on European railroads - the trolley system $1.5 \mathrm{kV}$ DC connection of output traction converters VSI is parallel ( $3 \mathrm{kV}$ DC trolley system is realized by serial connection).

\section{SINGLE PHASE MATRIX CONVERTER}

Figure 5 describes a detail of single-phase matrix converter MC. Four semiconductor switches are implemented as an "anti-serial" combination of two IGBT (see for example S0 and S1 in Fig. 5) or an anti-parallel combination of two IGCT, or reversible-blocking RB-IGBT.

Input terminals (in 1 -in2) are connected to a voltage source (capacitance $C_{f}$ of input filter) and output phases (out1-out2) are connected to a current source (leaking inductance of MFT or additional inductance $L_{k}$ for VSAR).

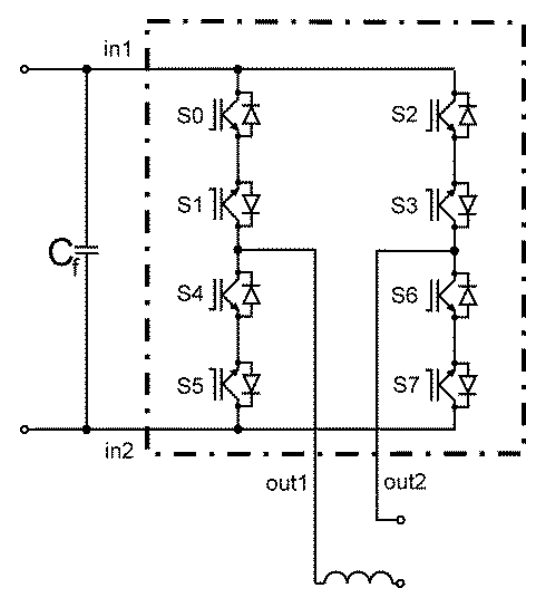

Fig. 5. Single-phase matrix converter.

Generally speaking, matrix converters can consist of $\mathbf{m}$-input terminals and $\mathbf{n}$-output phases (for three matrix converter $\mathbf{m}=\mathbf{n}=\mathbf{3}$ ). It is possible to describe actual switching combination using a matrix with $\mathbf{m}$ rows and $\mathbf{n}$ columns. Each element of this matrix can acquire "logical zero" (open switch) or "logical one" (closed switch).

Figure 6 shows switching combinations for a single phase matrix converter (according to Fig. $5 m=n=2$ ) described by only one scalar $(s=+1, s= \pm 0$ or $s=-1)$.

Switching combinations $s= \pm 0$ (Fig. 6(c) and Fig. 6(d)) give zero output voltage (so called "zero or NULL vectors") and create some unfavourable effects - e.g. oscillation and high voltage ripple in input filter.

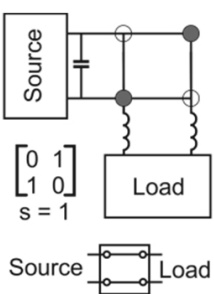

a)

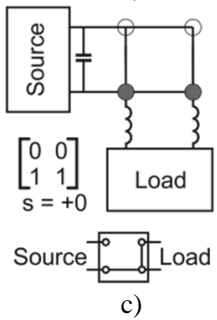

c)
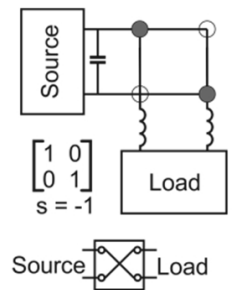

b)
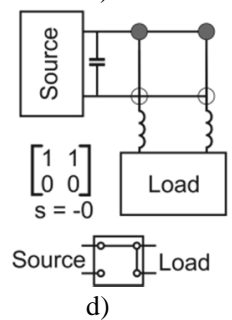

Fig. 6. Switching combination for single phase converter. 
Matrix converters are connected to a voltage-source at the input (filter capacitor) and to the current-source at the output (MFT leakage inductance). Therefore, the input phases cannot be short-circuited and the output phases cannot be disconnected.

In order to replace the matrix converter we have to implement a special control algorithm.

\section{MATRIX CONVERTER COMMUTATION}

The basic control algorithms employ a 4-step commutation based on knowledge of input voltage polarity (Fig. 7-Fig. 9) or output current polarity (Fig.10-Fig. 12). Practical realization can also be realized using its modification. Let us briefly describe the basic principles.

Figure 7 and Fig. 10 show matrix converters realized by IGCT designed to simplify the power circuits and the commutation explanation (the open switch is marked in black and the closed switch in white).

Commutation from switching state $s=+1$ to switching state $s=+O$ (for positive input voltage) is based on our knowledge of input voltage polarity as is described in Fig. 7.
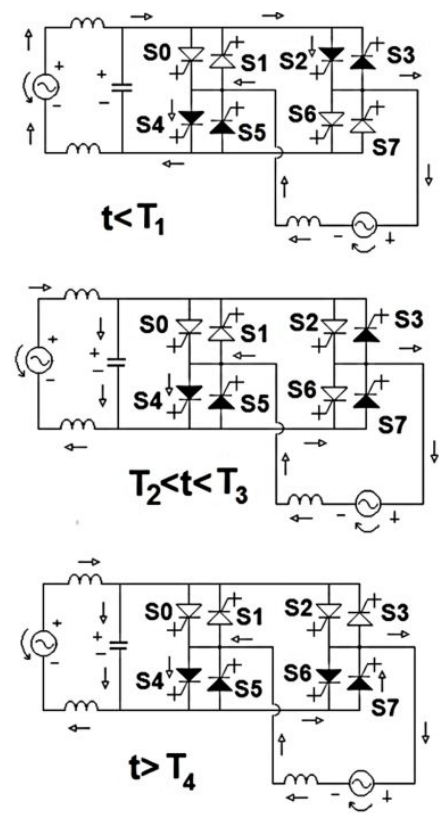

Fig. 7. Commutation by the knowledge of input voltage polarity (positive input voltage and positive output current).

Figure 8 presents the details of a switching sequence for the positive output current (the thick line means a closed device with current, the thin line means a closed device as well, but without current).

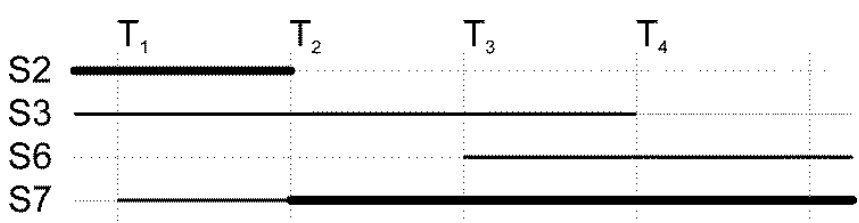

Fig. 8. Commutation by the knowledge of polarity of input voltage (positive input voltage and positive output current as in Fig. 7.).

Figure 9 shows the situation in Fig. 8 with opposite direction of output current. It is possible to use the same gate signals for commutation present in Fig. 8 in Fig. 9.

Error in input voltage polarity identification (low actual value of input voltage) should enable us to avoid the over- current - short circuit voltage input through open elements $\mathbf{S 3}$ and $\mathbf{S 7}$ in time intervals $\mathbf{t} \in\left(\mathbf{T}_{\mathbf{1}} ; \mathbf{T}_{\mathbf{4}}\right)$.

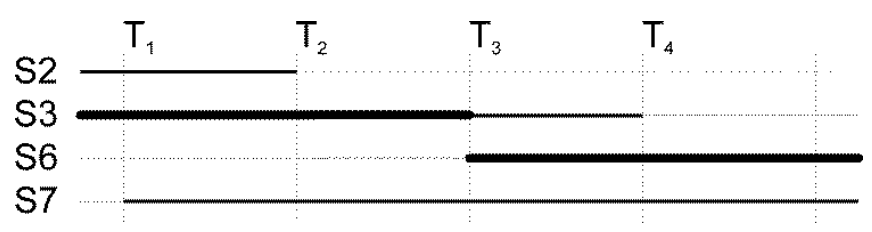

Fig. 9. Commutation according to the knowledge of polarity of input voltage (positive input voltage and negative output current).

The commutation algorithm is based on the knowledge of output current polarity as described in Fig. 10. Details of the switching sequence are shown in Fig. 11 (positive input voltage) and Fig. 12 (negative input voltage).
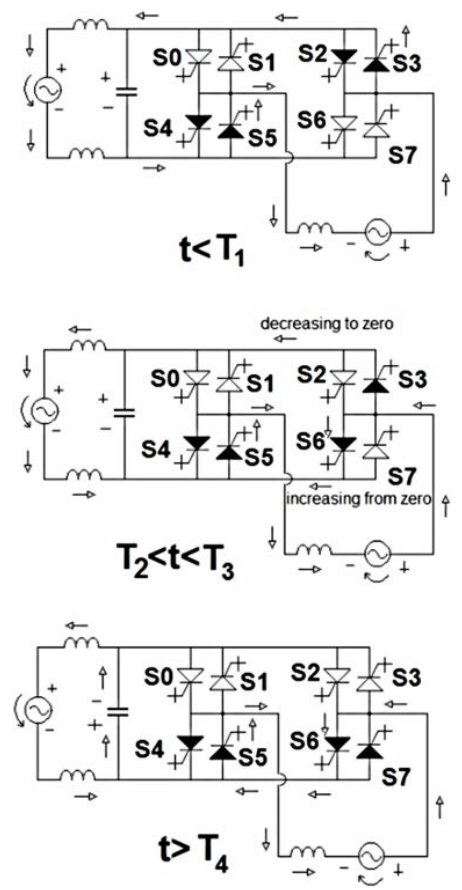

Fig. 10. Commutation according to the knowledge of polarity of output current (negative output current and positive input voltage).

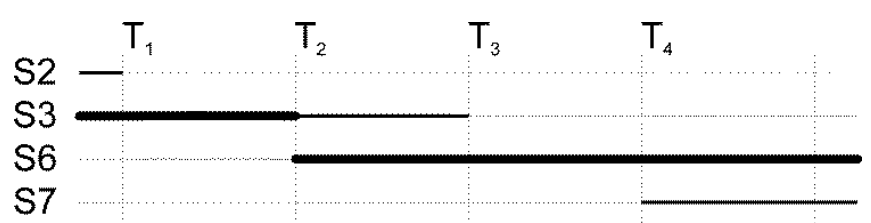

Fig. 11. Commutation based on the knowledge of polarity of output current (negative output current and positive input voltage as in Fig. 10).

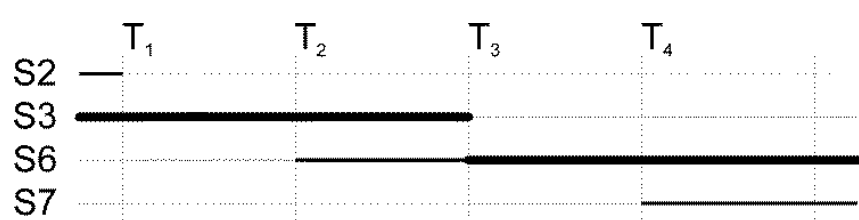

Fig. 12. Commutation based in the knowledge of polarity of output current (negative output current and negative input voltage).

Error in output current polarity identification (an actual low value of output current) causes the over-voltage on element $\mathbf{S 2}$ - disconnection of way for load current in time $\mathbf{T}_{1}$.

The above described basic commutation algorithms have a very different switching sequence and it is possible to combine them. Figure 13 shows the principle of combined algorithm (i.e. preferred method of commutation for actual 
values). In sections 2 and 8 there is a very small current, therefore we should firstly commutate based on our knowledge of input voltage polarity. In sections 4 and 6 (low voltage) we commutate based on our knowledge of output current polarity. In sections $1,3,7$ or 9 we can choose commutation based on current or voltage polarity knowledge.

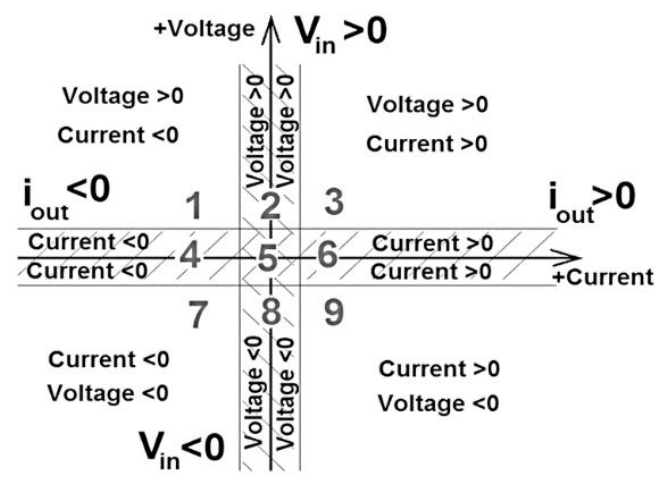

Fig. 13. Combined algorithms of commutation.

Centre section 5 is a problem, because both current and voltage are very low (polarity detection can cause a hazard).

\section{CONSTANT SWITCHING FREQUENCY OF MC}

Higher operating frequency causes the size minimisation of the transformer's iron core (reduction of transformer weight (Fig. 14)) for the same value of magnetic saturation.

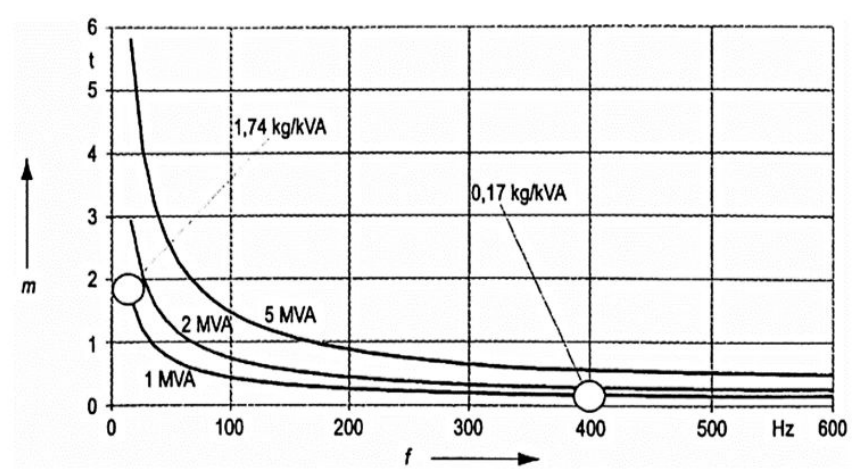

Fig. 14. Transformer weight as a function of switching frequency ([9]).

On other hand, very high frequency rapidly increases the voltage dips in MFT leakage inductances (watt-less leakage power factor) and additional power losses in converter (switching power losses), MFT (eddy currents losses, hysteresis losses) and all conductors (skin effect). We have to implement a special construction of MFT (special material of iron core).

\section{NOVEl CONTROL STRATEGY WITH OPTIMISATION OF SWITCHING FREQUENCY OF MC}

Matrix converter has a time-variable value of the magnitude of output voltage (Fig. 3). This means that constantly switching frequency is not optimal and we should adjust the actual value of switching frequency according to the actual value of output voltage in order to obtain maximal power with minimal switching frequency without saturating the magnetic core.

The actual value of induced voltage $u_{\text {ind }}$ in MFT is produced by magnetic flux $\Psi$

$$
u_{\text {ind }}=\frac{d \Psi}{d t} .
$$

It is valid for some time interval (2)

$$
d \Psi=\int_{t_{1}}^{t_{2}} u_{\text {ind }} d t
$$

Let us use some optimal "average value" of switching frequency $f_{S w A v}$ (synchronized with input trolley voltage). The number of switching instances during one period $T_{\text {Troll }}$ of supply trolley frequency is the ratio between these frequencies

$$
q=f_{\text {SwAv }} / f_{\text {Troll }}=T_{\text {Troll }} / T_{S w}=f_{S w} \times T_{\text {Troll }} .
$$

To minimize magnetic saturation we have to fix the same values of magnetic flux during each switching time interval. Let us use odd numbers (i.e. $j=1,3, \ldots$ ) for decreasing $\mathrm{mg}$. flux $\Psi$ (i.e. negative voltage in MFT) and even numbers (i.e. $j=2,4, \ldots$ ) for increasing it

$$
\begin{gathered}
-\Delta \Psi_{1, q}=\Delta \Psi_{2, q}=-\Psi_{3, q}=\Delta \Psi_{4, q}= \\
=-\Delta \Psi_{5, q}=\Delta \Psi_{6, q}=\cdots= \pm \Delta \Psi_{j, q}=\text { const } .
\end{gathered}
$$

For sinusoidal input voltage and actual switching combination $s(t)=s_{j}= \pm 1$ of MC we obtain for general $j=1,2,3,4, \ldots 2 q$

$$
\begin{gathered}
\Delta \Psi_{j, q}=\int_{t_{j-1, q}}^{t_{j, q}} u_{i n d} d t=\int_{t_{j-1, q}}^{t_{j, q}} U_{A} s(t) \times \sin (\omega \times t) d t= \\
=\int_{j-1, q}^{x_{j, q}} U_{A} s(x) \times \sin (x) \frac{d x}{\omega}=s_{j} \times \frac{U_{A}}{\omega} \times \int_{x_{j-1, q}}^{x_{j, q}} \sin (x) d x= \\
=s_{j} \times \frac{U_{A}}{\omega} \times\left[\cos \left(x_{j-1, q}\right)-\cos \left(x_{j, q}\right)\right] .
\end{gathered}
$$

Using time $\mathbf{t}_{\mathbf{1}, \mathbf{q}}$ for the first switching in maximal distance from the zero crossing reduces problems of commutation during zero crossing of input voltage and the first changing of polarity is realized by this zero crossing (without switching the MC)

$$
x_{0, q}=0
$$

For electric vehicle according to Fig. 1 (i.e. for low frequency transformer without MC $s=-1$ and $q=1$ )

$$
\begin{aligned}
\Delta \Psi_{1,1}= & -\frac{U_{A}}{\omega} \int_{x_{0,1}=0}^{x_{1,1}=\pi} \sin (x) d x=-\frac{U_{A}}{\omega}[\cos (0)-\cos (\pi)]= \\
& =-2 \times \frac{U_{A}}{\omega}=-T_{S w} \times \frac{U_{A}}{\pi}=-\frac{T_{\text {Troll }} \times U_{A}}{1 \times \pi} .
\end{aligned}
$$

MC enables an increase in frequency (i.e. ratio q) and reduces the value of magnetic flux saturation.

Decreasing the magnetic field in MFT (i.e. first odd numbers $j<q+1$, the switching combination of matrix 
converter $\mathrm{MC}$ is $s_{j}=-1$ )

$$
\Delta \Psi_{1, q}=\Delta \Psi_{3, q}=\Delta \Psi_{5, q}=\frac{\Delta \Psi_{1,1}}{q}=-\frac{T_{\text {Troll }} \times U_{A}}{q \times \pi} .
$$

Equations (8), (5) and (4) yield:

$$
\begin{gathered}
\Delta \Psi_{j, q}=-\frac{T_{\text {Troll }} \times U_{A}}{q \times \pi}=-\frac{T_{\text {Troll }} \times U_{A}}{2 \times \pi} \times \\
\times\left[\cos \left(x_{j-1, q}\right)-\cos \left(x_{j, q}\right)\right] \frac{1}{q}=\frac{\cos \left(x_{j-1, q}\right)-\cos \left(x_{j, q}\right)}{2} \\
\cos \left(x_{j, q}\right)=\cos \left(x_{j-1, q}\right)-\frac{2}{q}
\end{gathered}
$$

The first negative voltage-pulse begins (6) in position $x_{0, q}$ $=0$ and finish in time (10)

$$
x_{1, q}=\operatorname{arcos}\left(1-\frac{2}{q}\right)
$$

For other odd numbers we can obtain a general formula

$$
x_{j, q}=\operatorname{arcos}\left(1-\frac{2 \times j}{q}\right) \text {. }
$$

For increasing of magnetic flux in MFT (i.e. for the first even numbers $j<q+1$ the switching combination of MC is $\left.s_{j}=1\right)$

$$
\Delta \Psi_{2, q}=\Delta \Psi_{4, q}=\Delta \Psi_{6, q}=-\Delta \Psi_{1, q}=+\frac{T_{\text {Troll }} \times U_{A}}{\mathrm{q} \times \pi} .
$$

This result goes to a same result as in (10) and the final equation (12). General proof of (12) for all values (i.e. for $j=0,1,2,3,4, \ldots q, q+1)$ can be achieved by mathematical induction based equations (6), (11) and (10).

Figure 15 shows graphic examples for small ratio $\mathbf{q}$.

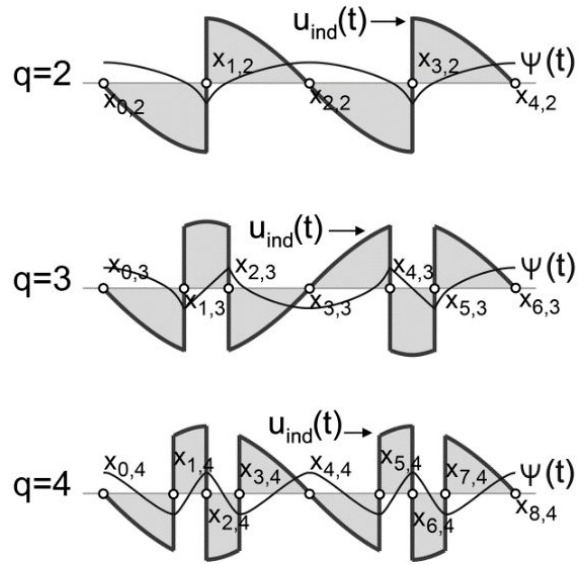

Fig. 15. Illuminated examples of voltage and $\mathrm{mg}$. flux in transformer for various values of average switching frequency.

\section{MODULAR CONCEPT}

Figure 16 describes the modular topology. Each MC has its own separate MFT with a single input and output. We have to apply a special control algorithm to balance the input voltages on input filter capacitors $\mathbf{C}_{\mathbf{f}}$. We can balance the input filter by:

a) inserting zero vectors in MCs,

b) phase shift between control signals of MCs,

c) additional control algorithm of VSAR in secondary side of (MFT).

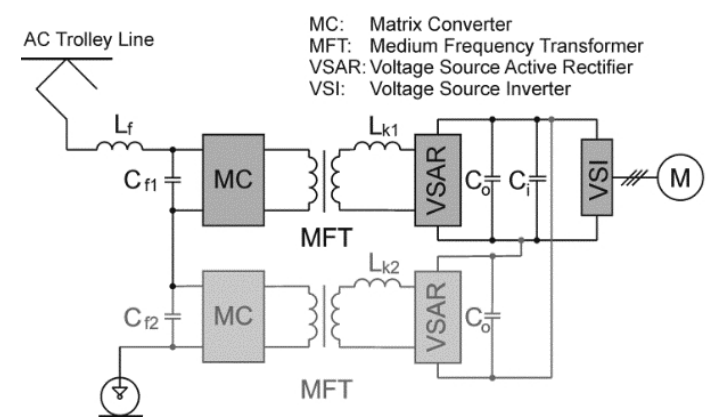

Fig. 16. Modular topology with middle frequency transformer MFT and matrix converters MC.

Control algorithms a) and b) adversely affect the input filter and can cause voltage ripples. Control algorithm c) enables quicker time-response (low voltage side with possibility to use higher switching frequency) and it allows smaller input filter.

\section{MEASUREMENT ON MATRIX CONVERTER}

Theoretical considerations of novel control strategy were verified by $\mathrm{PC}$ simulations and then compared with measurement on a laboratory model (Fig. 17). The power circuit of the laboratory model is realized according to Fig. 16. The control algorithm of the proposed traction converter topology was implemented into float DSP (TMS320F28335).

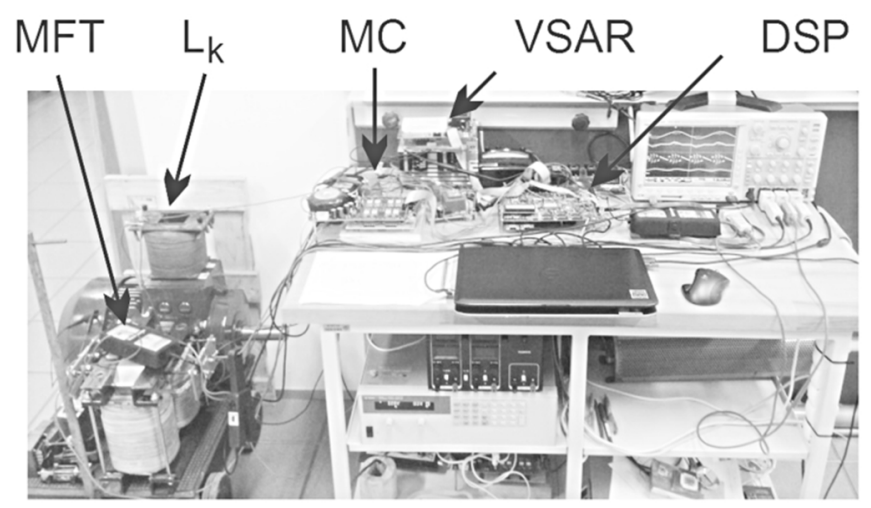

Fig. 17. Laboratory model of traction converter with middle frequency converter (MFT), input inductance $\mathrm{L}_{\mathrm{k}}$, matrix converter (MC) and voltagesource active rectifier (VSAR) controlled by DSP.

Figure 18-Fig. 20 show the input ("trolley") voltage $u_{\text {input }}$, input current $i_{\text {input }}$, input voltage of MFT $u_{M F T}$ and gate signals for IGBT in a matrix converter for different ratio $\mathbf{q}$. Real voltage has a small voltage ripple and oscillation (during commutation), because inductances in the output side of the matrix converter reduce the slow rate of current (it bring non-sinusoidal current and requirement to the input filter).

Figure 18 and Fig. 20 contain an even number of ratio $\mathbf{q}$ and it is evident that input voltage of MFT $u_{M F T}$ during the half period begins and ends with inverse polarity (e.g. begins as + voltage, ends as - voltage). 


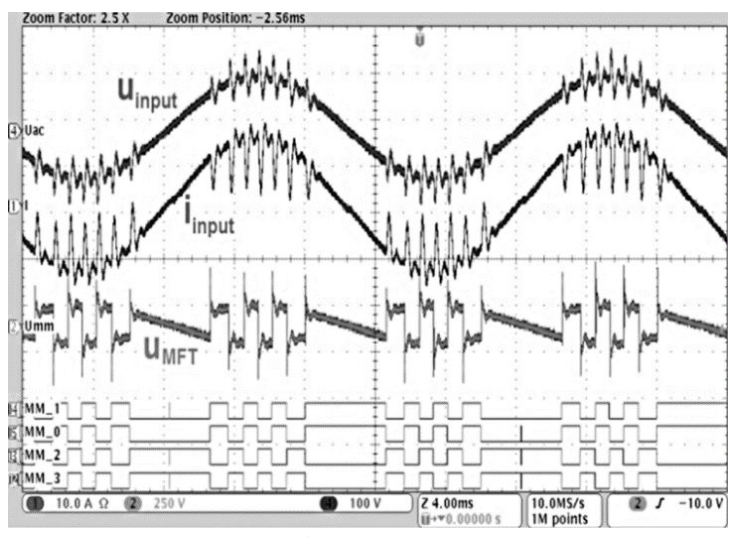

Fig. 18. Ratio $\mathrm{q}=8$ (input frequency $50 \mathrm{~Hz}$ and average switching frequency in MFT $400 \mathrm{~Hz}$ ).

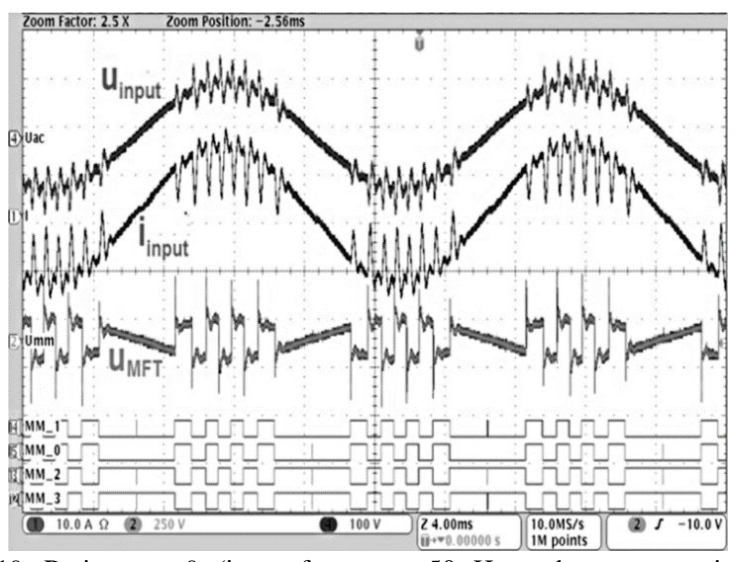

Fig. 19. Ratio $\mathrm{q}=9$ (input frequency $50 \mathrm{~Hz}$ and average switching frequency in MFT $450 \mathrm{~Hz}$ ).

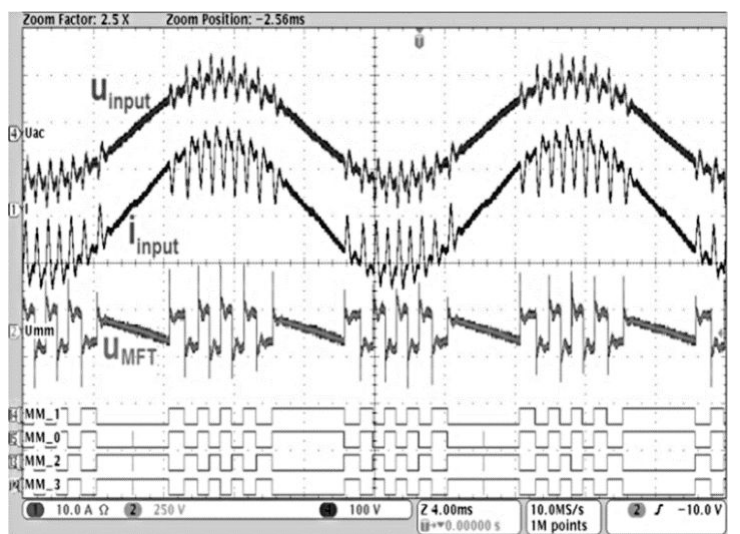

Fig. 20. Ratio $\mathrm{q}=10$ (input frequency $50 \mathrm{~Hz}$ and average switching frequency in MFT $500 \mathrm{~Hz}$ ).

Figure 19 presents an odd number of ratio $\mathbf{q}$ and input voltage of MFT $u_{M F T}$ during the half period begins and ends with the same polarity (e.g. begins as + voltage, ends as + voltage).

\section{CONCLUSIONS}

This article describes a novel control strategy for designing a traction converter for AC-Trolley Line traction vehicles which enables extensive transformer weight reduction. The traction converter consists of the input high voltage filter, input high voltage matrix converter, medium frequency transformer and output active rectifier.

The second and third chapters describe the matrix converter and the commutation method for the single-phase matrix converter. Commutation methods based on the knowledge of input voltage and output current polarity have been discussed in detail. The main focus is on uncertain states (near zero crossing, etc.).

The fourth and fifth chapters describe a novel control strategy of matrix converters with respect to magnetic saturation of MFTs. The focus is on relationships which produce optimized values of the switching angle in order to optimize the switching frequency of MC. The sixth chapter presents the new modular concept with several separate MFTs.

Theoretical considerations of novel control strategy were verified by PC simulations and compared with measurements in the laboratory model of traction converter.

\section{REFERENCES}

[1] X. She, R. Burgos, G. Wang, "Review of solid state transformer in the distribution system: from components to field application", IEEE Energy Conversion Congress and Exposition (ECCE), pp. 40774084, 2012.

[2] S. Kouro, M. Malinowski, K. Gopakumar, J. Pou, L. G. Franquelo, B. Wu, J. Rodriguez, M. A. Perez, J. I. Leon, "Recent advances and industrial applications of multilevel converters", IEEE Trans. on Ind. Electron., vol. 57, no. 8, pp. 2553-2580, 2010. [Online]. Available: http://dx.doi.org/10.1109/TIE.2010.2049719

[3] M. Glinka, R. Marquardt, "A new AC/AC multilevel converter family", IEEE Trans. Ind. Electron., vol. 52, no. 3, pp. 662-669, 2005. [Online]. Available: http://dx.doi.org/10.1109/TIE.2005.843973

[4] A. Rufer, N. Schibli, C. Chabert, C. Zimmermann, "Configurable front-end converters for multi current locomotives operated on $162 / 3$ $\mathrm{Hz} \mathrm{AC}$ and $3 \mathrm{kV}$ DC systems", IEEE Trans. on Power Electron. vol. 18, no. 5, pp. 1186-1193, 2003. [Online]. Available: http://dx.doi.org/10.1109/TPEL.2003.816191

[5] O. Deblecker, A. Moretti, F. Vallee, "Comparative study of softswitched isolated DC-DC converters for auxiliary railway supply", IEEE Trans. on Power Electron., vol. 23, no. 5, pp. 2218-2229, 2008 [Online]. Available: http://dx.doi.org/10.1109/TPEL.2008.2001879

[6] M. Glinka, "Prototype of multiphase modular-multilevel-converter with 2 MW power rating and 17- level- output voltage", 35th Annual IEEE Power Electronics Specialists Conf. Aachen, Germany 2004. [Online]. Available: http://dx.doi.org/10.1109/pesc.2004.1355234

[7] B. Engel, M. Victor, G. Bachmann, A. Falk, " 15 kV/16.7 Hz energy supply system with medium frequency transformer and 6,5 kV IGBTs in resonant operation", European Conf. Power Electronics and Applications (EPE), Toulouse, 2003.

[8] M. Steiner, H. Reinold, "Medium frequency topology in railway; power electronics and applications", European Conf. Power Electronics and Applications (EPE), Aalborg, 2007.

[9] M. Victor, "Energieumwandlung auf AC-Triebfahrzeugen mit mittelfrequenztransformator", Fahrzeugtechnikeb 103 Heft 11, 2005, pp. 505-510. (in German)

[10] D. Dujic, Ch. Zhao, A. Mester, J. K. Steinke, M. Weiss, S. LewdeniSchmid, T. Chaudhuri, P. Stefanutti, "Power electronic traction transformer-lowvoltage prototype", IEEE Trans. Power electronics, vol. 28, no. 12, pp. 5522-5534, 2013. [Online]. Available: http://dx.doi.org/10.1109/TPEL.2013.2248756

[11] N. Hugo, P. Stefanutti, M. Pellerin, A Akdag, "Power electronics traction transformer", European Conf. Power Electronics and Applications, 2007, pp.1-10. [Online]. Available: http://dx.doi.org/10.1109/epe.2007.4417649

[12] C. Aghion, M. Lucanu, O. Ursaru, N. Lucanu, "Direct AC-AC stepdown single-phase converter with improved performances", Elektronika Ir Elektrotechnika, vol. 18, no. 10, pp. 33-36, 2012. [Online]. Available: http://dx.doi.org/10.5755/j01.eee.18.10.1305

[13] J. W. Kolar, T. Friedli, J. Rodriguez, P. W. Wheeler, "Review of three-phase PWM AC-AC converter topologies", IEEE Trans. Ind. Electron., vol. 58, pp. 4988-5006, 2011. [Online]. Available: http://dx.doi.org/10.1109/TIE.2011.2159353

[14] E. Karaman, M. Farasat, A. M. Trzynadlowski, "A comparative study of series and cascaded Z-source matrix converters", IEEE Trans. Ind. Electron., vol. 61, no. 10, pp. 5164-5173, 2014. [Online]. Available: http://dx.doi.org/10.1109/TIE.2014.2301766

[15] F. Gruson, P. Le Moigne, P. Delarue, A. Videt, X. Cimetiere, M. Arpilliere, "A simple carrier-based modulation for the SVM of the matrix converter", IEEE Trans. Ind. Informatics, vol. 9, no. 2, pp. 947-956, 2013. [Online]. Available: http://dx.doi.org/10.1109/ TII.2012.2224354 